\title{
An inequality for the Selberg zeta-function, associated to the compact Riemann surface
}

\author{
Igoris Belovas
}

\begin{abstract}
We consider the absolute values of the Selberg zeta-function, associated to the compact Riemann surface, at places symmetric with respect to the line $\Re(s)=1 / 2$. We prove an inequality for the Selberg zetafunction, extending the result of R. Garunkštis and A. Grigutis.
\end{abstract}

\section{Introduction}

Let $s=\sigma+i t$ be a complex variable and $\zeta(s)$ be the Riemann zeta-function. T. S. Trudgian [6] obtained, that

$$
|\zeta(1-s)|>|\zeta(s)|
$$

except at the zeros of $\zeta(s)$, with $|t| \geqslant 6.29073$ and $\sigma>1 / 2$. By well-known functional equation for the Riemann zeta-function,

$$
\zeta(s)=2^{s} \pi^{s-1} \sin \frac{\pi s}{2} \Gamma(1-s) \zeta(1-s),
$$

$\zeta(1-s)$ and $\zeta(s)$ have the same zeros when $0<\sigma<1$. Inequalities of (1) type are of great interest, since a necessary and sufficient condition for the Riemann hypothesis is $|\zeta(1-s)|>|\zeta(s)|$, where $\sigma>1 / 2$ and $|t| \geqslant 6.29073$.

Key Words: Inequalities, Selberg zeta-function, Clausen function.

2010 Mathematics Subject Classification: Primary 11M36; Secondary 30A10.

Received: 17.12 .2018

Revised: 10.03 .2019

Accepted: 15.03.2019 
Garunkštis and Grigutis examined, whether Selberg zeta-function, associated to the compact Riemann surface and the modified Selberg zeta-function satisfy the inequalities of (1) type (see Theorems 1 and 2 in [2]). Let $\mathbb{H}$ be the upper half-plane and $\Gamma$ be a subgroup of $\operatorname{PSL}(2, \mathbb{R})$. Let $\Gamma \backslash \mathbb{H}$ be a compact Riemann surface of genus $g \geqslant 2$. The Selberg zeta-function, associated to the compact Riemann surface of genus $g \geqslant 2$, is defined as follows $[2,5]$

$$
Z_{C}(s)=\prod_{\{P\}} \prod_{k=0}^{\infty}\left(1-N(P)^{-s-k}\right),
$$

where $\sigma>1$ and $\{P\}$ runs trough all the primitive hyperbolic conjugacy classes of $\Gamma$ and $N(P)=\alpha^{2}$ if the eigenvalues of $P$ are $\alpha$ and $\alpha^{-1}(|\alpha|>1)$. The Selberg zeta-function has a meromorphic continuation to $\mathbb{C}[5]$.

The Selberg zeta-function, associated to the compact Riemann surface, satisfies (cf. (2)) [2, 4] the functional equation

$$
Z_{C}(s)=Z_{C}(1-s) \exp \left(4 \pi(g-1) \int_{0}^{s-1 / 2} \theta \tan \pi \theta d \theta\right) .
$$

Garunkštis and Grigutis have proved [2], that for $\sigma>1 / 2$ and $t \geqslant 0.361$,

$$
\left|Z_{C}(1-s)\right|>\left|Z_{C}(s)\right|
$$

In this research we extend the result of R. Garunkštis and A. Grigutis. We apply the technics of estimation used in [1].

\section{An inequality for the Selberg zeta-function, associated to the compact Riemann surface}

Theorem 1. Let $Z_{C}(s)$ be the Selberg zeta-function associated to the compact Riemann surface of genus $g \geqslant 2$. Then, for $\sigma>1 / 2$ and $t \geqslant t_{0}$ we have

$$
\left|Z_{C}(1-s)\right|>\left|Z_{C}(s)\right| \text {. }
$$

Here $t_{0}=0.165 \ldots$.

By the functional equation for the Selberg zeta-function (3) we have

$$
\log \left|\frac{Z_{C}(s)}{Z_{C}(1-s)}\right|=\underbrace{(g-1)}_{>0} \Re(Q(s)),
$$

here

$$
Q(s)=4 \pi \int_{0}^{s-1 / 2} v \tan \pi v \mathrm{~d} v
$$


Integral (6) can be evaluated using triangular contour with vertices at $A(0,0)$, $B(\sigma-1 / 2, t)$ and $C(\sigma-1 / 2,0): \int_{A C}+\int_{C B}+\int_{B A}=0$. Hence,

$$
\underbrace{\Re(Q(s))}_{\Re \int_{A B}}=\underbrace{I_{1}(\sigma)}_{\int_{A C}}+\underbrace{R(\sigma, t)}_{\Re \int_{C B}} \text {. }
$$

Here

$$
I_{1}(\sigma)=4 \pi \int_{0}^{\sigma-1 / 2} \theta \tan \pi \theta \mathrm{d} \theta
$$

and

$$
R(\sigma, t)=\Re\left\{\int_{0}^{t} i(\sigma-1 / 2+i \theta) \tan (\pi(\sigma-1 / 2+i \theta)) d \theta\right\} .
$$

Calculating function $I_{1}(\sigma)(8)$, we obtain

$$
I_{1}(\sigma)=-\frac{2}{\pi} \mathrm{Cl}_{2}(2 \pi \sigma)-2(2 \sigma-1) \log |2 \sin \pi \sigma| .
$$

Here $\mathrm{Cl}_{2}(x)$ is the Clausen function of order 2,

$$
\mathrm{Cl}_{2}(x)=-\int_{0}^{x} \log \left|2 \sin \frac{t}{2}\right| \mathrm{d} t .
$$

Calculate function $R(\sigma, t)(9)$, we obtain

$$
R(\sigma, t)=\underbrace{\int_{0}^{t} \frac{4 \pi \theta \sin 2 \pi \sigma}{\cosh 2 \pi \theta-\cos 2 \pi \sigma} \mathrm{d} \theta}_{=I_{2}(\sigma, t)}+\underbrace{\int_{0}^{t} \frac{4 \pi(1 / 2-\sigma) \sinh 2 \pi \theta}{\cosh 2 \pi \theta-\cos 2 \pi \sigma} \mathrm{d} \theta}_{=I_{3}(\sigma, t)}
$$

Note that $R(\sigma, t)$ is even function by $t$, thus, it suffices to consider positive $t$ values.

Calculating $I_{2}(\sigma, t)$, we obtain (cf. 3.531.8 in [3]), that

$$
\begin{aligned}
I_{2}(\sigma, t) & =4 \pi \sin 2 \pi \sigma \int_{0}^{t} \frac{\theta}{\cosh 2 \pi \theta-\cos 2 \pi \sigma} \mathrm{d} \theta \\
& =\frac{2}{\pi}(\Lambda(u(\sigma, t)+\pi \sigma)-\Lambda(u(\sigma, t)-\pi \sigma)-2 \Lambda(\pi \sigma))= \\
& =\frac{1}{\pi} \mathrm{Cl}_{2}(2 u(\sigma, t)+2 \pi \sigma+\pi)-\frac{1}{\pi} \mathrm{Cl}_{2}(2 u(\sigma, t)-2 \pi \sigma+\pi)- \\
& \left.-\frac{2}{\pi} \mathrm{Cl}_{2}(2 \pi \sigma+\pi)\right) .
\end{aligned}
$$

Here $\Lambda(x)$ is the Lobachevsky function,

$$
\Lambda(x)=-\int_{0}^{x} \log |\cos t| \mathrm{d} t=\mathrm{Cl}_{2}(2 x+\pi) / 2+x \log 2,
$$


and

$$
u(\sigma, t)=\arctan (\tanh \pi t \cot \pi \sigma) .
$$

Calculating $I_{3}(\sigma, t)$, we obtain

$$
\begin{aligned}
I_{3}(\sigma, t)= & -(2 \sigma-1) \log (\cosh 2 \pi t-\cos 2 \pi \sigma)+ \\
& +2(2 \sigma-1) \log |\sin \pi \sigma|+(2 \sigma-1) \log 2,
\end{aligned}
$$

Let us denote

$$
L(\sigma, t)=\Re(Q(s)) .
$$

In view of (5), to prove the statement of the theorem, it is enough to show that, for $\sigma>1 / 2$ and $t \geqslant t_{0}$ the function $L(\sigma, t)$ is negative. By (7) and (10)-(15), we have

$$
\begin{aligned}
L(\sigma, t)= & -\frac{2}{\pi} \mathrm{Cl}_{2}(2 \pi \sigma)+4 \pi \sin 2 \pi \sigma \int_{0}^{t} \frac{\theta}{\cosh 2 \pi \theta-\cos 2 \pi \sigma} \mathrm{d} \theta- \\
& -(2 \sigma-1) \log (\cosh 2 \pi t-\cos 2 \pi \sigma)-(2 \sigma-1) \log 2 .
\end{aligned}
$$

Taking into account the duplication formula for the Clausen function,

$$
\mathrm{Cl}_{2}(2 \theta)=2 \mathrm{Cl}_{2}(\theta)-2 \mathrm{Cl}_{2}(\pi-\theta),
$$

and the properties of the Clausen function,

$$
\begin{aligned}
& \mathrm{Cl}_{2}(\theta)=\mathrm{Cl}_{2}(\theta+2 \pi m), \quad m \in \mathbb{Z}, \\
& \mathrm{Cl}_{2}(\theta)=-\mathrm{Cl}_{2}(-\theta) .
\end{aligned}
$$

we obtain

$$
\begin{aligned}
L(\sigma, t) & =\frac{1}{\pi} \mathrm{Cl}_{2}(2 u(\sigma, t)+2 \pi \sigma+\pi)- \\
& -\frac{1}{\pi} \mathrm{Cl}_{2}(2 u(\sigma, t)-2 \pi \sigma+\pi)-\frac{1}{\pi} \mathrm{Cl}_{2}(4 \pi \sigma)- \\
& -(2 \sigma-1) \log (\cosh 2 \pi t-\cos 2 \pi \sigma)-(2 \sigma-1) \log 2 .
\end{aligned}
$$

Next we establish a lemma concerning the behaviour of the derivative of the function $L(\sigma, t)$.

Lemma 1. For $\sigma>1 / 2$ and $t>0$, the derivative $L_{t}^{\prime}(\sigma, t)$ is negative.

Proof. Let us calculate the first partial derivative. By (7) and (12) we have

$$
\begin{aligned}
L_{t}^{\prime}(\sigma, t) & =\frac{4 \pi}{\cosh 2 \pi t-\cos 2 \pi \sigma}+\frac{4 \pi(1 / 2-\sigma) \sinh 2 \pi t}{\cosh 2 \pi t-\cos 2 \pi \sigma} \\
& =\underbrace{\frac{4 \pi}{\cosh 2 \pi t-\cos 2 \pi \sigma}}_{>0} \underbrace{(t \sin 2 \pi \sigma+(1 / 2-\sigma) \sinh 2 \pi t)}_{=N(\sigma, t)} .
\end{aligned}
$$


For $\sigma \in(n-1 / 2, n), n \in \mathbb{N}$, we have $N(\sigma, t)<0$ (since $\sin 2 \pi \sigma$ is negative) For $\sigma \in(n, n+1 / 2), n \in \mathbb{N}$,

$$
N(\sigma, t)<t-\frac{1}{2} \sinh 2 \pi t<0 .
$$

For $\sigma=n, n \in \mathbb{N}$, we have $N(\sigma, t)=(1 / 2-n) \sinh 2 \pi t<0$.

Thus, for $\sigma>1 / 2$ and $t>0$, the function $N(\sigma, t)$ is negative, yielding us (cf. (19)) $L_{t}^{\prime}(\sigma, t)<0$.

Let us denote

$$
\begin{aligned}
B_{0}(\sigma) & =\frac{1}{\pi} \mathrm{Cl}_{2}\left(2 u\left(\sigma, t_{0}\right)+2 \pi \sigma+\pi\right)- \\
& -\frac{1}{\pi} \mathrm{Cl}_{2}\left(2 u\left(\sigma, t_{0}\right)-2 \pi \sigma+\pi\right)-\frac{1}{\pi} \mathrm{Cl}_{2}(4 \pi \sigma) .
\end{aligned}
$$

Lemma 2. The function $B_{0}(\sigma)$ is

(i) periodic with period $P=1$, thus $B_{0}(\sigma)=B_{0}(\sigma+m), m \in \mathbb{Z}$,

(ii) odd, $B_{0}(\sigma)=-B_{0}(-\sigma)$,

(iii) bounded, $\left|B_{0}(\sigma)\right| \leqslant C_{0}$.

Here $C_{0}=0.46342 \ldots$.

Proof. The function $u\left(\sigma, t_{0}\right)(14)$ is periodic by $\sigma$ with period $P=1$ and the Clausen function $\mathrm{Cl}_{2}(\theta)$ is periodic with period $P=2 \pi$, hence the first statement of the lemma. Note that $\lim _{\sigma \rightarrow 1} B_{0}(\sigma)=0$. Next, the Clausen function (of order 2) and the function $u\left(\sigma, t_{0}\right)$ are odd, yielding us the second statement of the lemma.

In view of (i) and (ii) it is enough to consider the function $B_{0}(\sigma)$ in the interval $\sigma \in(1 / 2,1)$. Note that $B_{0}(1 / 2)=B_{0}(1)=0$. Calculating derivatives of $B_{0}(\sigma)$, we obtain

$$
B_{0}^{\prime}(\sigma)=-\frac{4 \pi t_{0} \sinh 2 \pi t_{0}}{\cosh 2 \pi t_{0}-\cos 2 \pi \sigma}+2 \log \left(\cosh 2 \pi t_{0}-\cos 2 \pi \sigma\right)+2 \log 2 .
$$

and

$$
B_{0}^{\prime \prime}(\sigma)=4 \pi \underbrace{\sin 2 \pi \sigma}_{<0} \underbrace{\left(\frac{2 \pi t_{0} \sinh 2 \pi t_{0}}{\left(\cosh 2 \pi t_{0}-\cos 2 \pi \sigma\right)^{2}}+\frac{1}{\cosh 2 \pi t_{0}-\cos 2 \pi \sigma}\right)}_{>0} .
$$

Let us denote

$$
\omega_{0}(\sigma)=\frac{1}{\cosh 2 \pi t_{0}-\cos 2 \pi \sigma}
$$


and

$$
a_{0}=4 \pi t_{0} \sinh 2 \pi t_{0} .
$$

Hence, by $(21)$, solving the equation $B_{0}^{\prime}\left(\sigma_{0}\right)=0$, we obtain

$$
-a_{0} \omega_{0}-2 \log \omega_{0}+\log 4=0 \quad \Longrightarrow \quad \omega_{0}=2 W\left(a_{0}\right) / a_{0} .
$$

Here $W(x)$ is the Lambert $W$ function. Thus,

$$
\cos 2 \pi \sigma_{0}=\cosh 2 \pi t_{0}-\frac{2 \pi t_{0} \sinh 2 \pi t_{0}}{W\left(4 \pi t_{0} \sinh 2 \pi t_{0}\right)},
$$

and

$$
\sigma_{0}=1-\frac{1}{2 \pi} \arccos \left(\cosh 2 \pi t_{0}-\frac{2 \pi t_{0} \sinh 2 \pi t_{0}}{W\left(4 \pi t_{0} \sinh 2 \pi t_{0}\right)}\right)=0.79336 \ldots
$$

Note that $B_{0}^{\prime \prime}(\sigma)(22)$ is negative for $\sigma \in(1 / 2,1)$, hence $B_{0}\left(\sigma_{0}\right)=0.46342 \ldots$ is the maxima of the function, yielding us the third statement of the lemma.

\section{Proof of Theorem 1}

Proof. By Lemma 1, it is enough to prove the theorem for fixed $t=t_{0}$. Let us denote $L_{0}(\sigma)=L\left(\sigma, t_{0}\right)$.

First consider the function $L_{0}(\sigma)$ for $\sigma \geqslant \sigma_{1}>1 / 2$. By (18) and Lemma 2, we have

$$
\begin{aligned}
L_{0}(\sigma) & <C_{0}-(2 \sigma-1) \log \left(\cosh 2 \pi t_{0}-\cos 2 \pi\right)-(2 \sigma-1) \log 2= \\
& =C_{0}-4(\sigma-1 / 2) \log \left(2 \sinh \pi t_{0}\right) .
\end{aligned}
$$

Hence, $L_{0}(\sigma)<0$ for $\sigma \geqslant \sigma_{1}$. Here

$$
\sigma_{1}=\frac{1}{2}+\frac{C_{0}}{4 \log \left(2 \sinh \pi t_{0}\right)}=1.94001 \ldots
$$

Next, consider the function $L_{0}(\sigma)$ for $1 / 2<\sigma<\sigma_{1}$. Calculating derivatives of the function, we obtain (cf. (18), (20) and (21)), that

$$
\begin{aligned}
L_{0}^{\prime}(\sigma)= & \underbrace{\frac{-4 \pi}{\cosh 2 \pi t_{0}-\cos 2 \pi \sigma}}_{<0} \underbrace{\left(d_{0}+(\sigma-1 / 2) \sin 2 \pi \sigma\right)}_{=f_{0}(\sigma)}, \\
L_{0}^{\prime \prime}(\sigma)= & -4 \pi \frac{\sin 2 \pi \sigma+2 \pi(\sigma-1 / 2) \cos 2 \pi \sigma}{\cosh 2 \pi t_{0}-\cos 2 \pi \sigma}+ \\
& +\frac{8 \pi^{2} \sin 2 \pi \sigma}{\left(\cosh 2 \pi t_{0}-\cos 2 \pi \sigma\right)^{2}} f_{0}(\sigma) .
\end{aligned}
$$


Here $d_{0}=t_{0} \sinh 2 \pi t_{0}=0.203 \ldots$.

The function $f_{0}(\sigma)$ has three zeros in the interval $\left(1 / 2, \sigma_{1}\right)$.

Indeed, for $\sigma \in(1 / 2,3 / 4)$, the derivative $f_{0}^{\prime}(\sigma)$ is negative, while $f_{0}(1 / 2)>$ 0 and $f_{0}(3 / 4)<0$. Hence, the first root $\hat{\sigma}_{1} \in(1 / 2,3 / 4)$. Note that $L_{0}^{\prime \prime}\left(\hat{\sigma}_{1}\right)>0$ (cf. $(24))$.

For $\sigma \in(3 / 4,1)$, the second derivative $f_{0}^{\prime \prime}(\sigma)$ is positive (hence the function is convex in the interval), while $f_{0}(3 / 4)<0$ and $f_{0}(1)>0$. Thus, the second root $\hat{\sigma}_{2} \in(3 / 4,1)$. Calculating numerically, we obtain $\hat{\sigma}_{2}=0.919 \ldots$ and $L_{0}^{\prime \prime}\left(\hat{\sigma}_{2}\right)<0$.

For $\sigma \in(1,3 / 2)$, the function $f_{0}(\sigma)$ is positive, hence no zeros in the interval.

For $\sigma \in(3 / 2,7 / 4)$, the derivative $f_{0}^{\prime}(\sigma)$ is negative, while $f_{0}(3 / 2)>0$ and $f_{0}(7 / 4)<0$. Hence, the third root $\hat{\sigma}_{3} \in(3 / 2,7 / 4)$. Note that $L_{0}^{\prime \prime}\left(\hat{\sigma}_{3}\right)>0$ (cf. $(24))$.

For $\sigma \in\left(7 / 4, \sigma_{1}\right)$, the second derivative $f_{0}^{\prime \prime}(\sigma)$ is positive (hence the function is convex in the interval), while $f_{0}(7 / 4)<0$ and $f_{0}\left(\sigma_{1}\right)<0$, hence no zeros in the interval.

The only maxima of the function $L_{0}(\sigma)$ for $1 / 2<\sigma<\sigma_{1}$ corresponds $\hat{\sigma}_{2}$. However, $L_{0}\left(\hat{\sigma}_{2}\right)$ is negative, yielding us the statement of the theorem.

Remark 1. Let us denote for $\sigma \in(n+1 / 2, n+1), n \in \mathbb{N}_{0}$,

$$
\varphi_{n}(\sigma)=\{t \mid L(\sigma, t)=0\} .
$$

Then

$$
t_{0}=\max _{1 / 2<\sigma<1} \varphi_{0}(\sigma)
$$

Calculating $t_{0}$ numerically, we obtain optimal $t_{0}=0.165 \ldots$.

\section{References}

[1] I. Belovas And L. Sakalauskas, An inequality for the modulus of the ratio of two complex gamma functions, Miskolc Math. Notes, 20, 1 (2019), $115-130$.

[2] R. GarunKštis And A. Grigutis, The size of the Selberg zeta-function at places symmetric with respect to the line $\operatorname{Re}(s)=1 / 2$, Results. Math., 70, 1 (2016), 271-281.

[3] I. S. Gradshteyn And I. M. Ryzhik, Table of Integrals, Series, and Products, 8th edition ed. Academic Press, 2014.

[4] D. A. Hejhal, The Selberg trace formula for PSL(2, $\mathbb{R})$ vol. 1. Lecture Notes in Mathematics, vol. 548. Springer, Berlin (1976). 
AN INEQUALITY FOR THE SELBERG ZETA-FUNCTION, ASSOCIATED

[5] D. A. Hejhal, The Selberg trace formula for $\operatorname{PSL}(2, \mathbb{R})$, vol. 2. Lecture Notes in Mathematics, vol. 1001. Springer, Berlin (1983)

[6] T. S. Trudgian, A short extension of two of Spiras results, J. Math. Inequal., 80, 3-4 (2015), 795-798.

Igoris BELOVAS,

Institute of Data Science and Digital Technologies,

Vilnius University,

Akademijos str. 4, LT-04812 Vilnius, Lithuania.

Email: Igoris.Belovas@mii.vu.lt 